\title{
Approximate Inverse Meets Local Tomography
}

\author{
Andreas Rieder ${ }^{1, \dagger}, *$ Rainer Dietz ${ }^{2, \ddagger}$ and Thomas Schuster ${ }^{2, \S}$ \\ ${ }^{1}$ Institut für Wissenschaftliches Rechnen und Mathematische Modellbildung (IWRMM), \\ Universität Karlsruhe, 76128 Karlsruhe, Germany \\ ${ }^{2}$ Fachbereich Mathematik, Geb. 36, Universität des Saarlandes, 66041 Saarbrücken, \\ Germany
}

Communicated by R. Kress

Local or lambda tomography reconstructs $\Lambda f$ which has the same discontinuities as the searched-for density distribution $f$. Computing $\Lambda f$, however, requires only local tomographic measurements. Local tomography is usually implemented by a filtered backprojection algorithm (FBA). In the present article we design reconstruction filters for the FBA such that $\Lambda^{2 m+1} f$ will be reconstructed for a given $m \in \mathbb{N}_{0}$. Moreover, we prove convergence and convergence rates for the FBA as the discretization step size goes to zero. To this end we express the FBA in the framework of approximate inverse. Based on our analysis we further propose a scheme which yields a proper scaling of the reconstruction filters. Numerical experiments illustrate the analytic results. Copyright (C) 2000 John Wiley \& Sons, Ltd.

KEY WORDS: approximate inverse; local tomography; lambda tomography; filtered backprojection

\section{Introduction}

Local tomography recovers jump discontinuities of the searched-for density distribution $f$ in a point $x$ using only integrals of lines passing near to $x$. Typical applications for local tomography are non-destructive testing and local microtomography.

The analytic basis of lambda tomography in $2 \mathrm{D}$ is the formula

$$
\Lambda^{-\alpha} f=(2 \pi)^{-1} \mathbf{R}^{*} \Lambda^{1-\alpha} \mathbf{R} f
$$

where $\mathbf{R}$ denotes the Radon transform mapping a function to its line integrals and $\mathbf{R}^{*}$ is the adjoint with respect to a suitable $L^{2}$-space (see e.g. Natterer [14]). Formally, $\Lambda$ is the square root of the Laplacian $-\Delta: \Lambda=(-\Delta)^{1 / 2}$. Note that any positive even power of $\Lambda$ is a local operator in the following sense: to compute $\Lambda^{2 \beta} f(x)$ we only need to know the values of $f$ near $x$. On the contrary, any positive odd power of $\Lambda$ is a global integral operator.

\footnotetext{
* Correspondence to: A. Rieder, Institut für Wissenschaftliches Rechnen und Mathematische Modellbildung (IWRMM), Universität Karlsruhe, 76128 Karlsruhe, Germany

${ }^{\dagger}$ E-mail: andreas.rieder@math.uni-karlsruhe.de

‡E-mail: rainer@num.uni-sb.de

${ }^{\S}$ E-mail: thomas@num.uni-sb.de
}

Contract grant sponsor: Deutsche Forschungsgemeinschaft; contract grant no: Lo310/4-1. 
With this knowledge let us have a closer look at (1.1). For $\alpha=0$ we have standard tomography. Here, the global operator $\Lambda$ acts on the data $\mathbf{R} f$. Reconstructing $f$ in $x$ therefore calls for all data (even integrals of $f$ over lines 'far away' from $x$ are needed).

Now set $\alpha=-1$. Thus, (1.1) represents a local reconstruction formula for $\Lambda f$. This situation is usually referred to as local tomography. Since $\Lambda f$ and $f$ share the same singular support, both functions have the same discontinuities. So, if we are interested in edges only, $\Lambda f$ tells us the story at much less computational expense than in standard tomography.

The idea of reconstructing $\Lambda f$ is due to Smith and Keinert [18] as well as Vainberg and Faingois [20]. Analytic properties of $\Lambda$ have been studied extensively by Faridani et al. $[6,5]$. In particular, they propose techniques for cup removing and estimating the sizes of density jumps from local data (see also Katsevich and Ramm [8]).

An algorithmic realization of local tomography may be based on the formula ( $\star$ denotes an appropriate convolution)

$$
f \star e=\mathbf{R}^{*}(v \star \mathbf{R} f), \quad e=\mathbf{R}^{*} v
$$

where $f \star e=\Lambda f \star \tilde{e}$ and $\tilde{e}$ is a smooth approximation to the $\delta$-distribution. The computation of the smoothed (mollified) version $\Lambda f \star \tilde{e}$ rather than $\Lambda f$ has a stabilizing (regularizing) effect.

In this paper we pursue two objectives. First, we investigate which properties of a compactly supported $v$ lead to an $e$ such that $f \star e(x) \approx \Lambda^{2 m+1} f(x)$ for an $m \in \mathbb{N}_{0}$ (section 3). We also propose a scheme to design such functions $v$ with a prescribed smoothness. Second, we discretize (1.2) yielding a variation of the filtered backprojection algorithm, (see e.g. Natterer [14, Chapter V]). For this algorithm we show convergence to $\Lambda^{2 m+1} f$ as the discretization step size approaches zero (section 4 ). Moreover, our convergence analysis tells us how to scale $v$ and $e$ properly for a fixed discretization step size. Numerical experiments for $m=0$ and 1 illustrate some of our results in section 5 .

Our investigations will be described in the framework of approximate inverse (see Louis and Maass [12]). Thus, we can rely on our convergence theory developed in [16]. The present paper may be viewed as a sequel to [16].

We start our exposition in the next section by introducing some notation and by commenting briefly on the concept of approximate inverse. For a more general investigation of approximate inverse, see Louis $[10,11]$.

\section{Tomographic reconstruction as approximate inverse}

The Radon transform $\mathbf{R}$ maps a function $f \in L^{2}(\Omega)$ to its line integrals. Here, $\Omega$ is the unit ball in $\mathbb{R}^{2}$ centered at the origin. We have that

$$
\mathbf{R} f(s, \vartheta):=\int_{L(s, \vartheta) \cap \Omega} f(x) \mathrm{d} \sigma(x)
$$

The lines are parameterized by $L(s, \vartheta)=\left\{\tau \omega^{\perp}(\vartheta)+s \omega(\vartheta) \mid \tau \in \mathbb{R}\right\}$ where $\left.s \in\right]-1,1[$, $\omega(\vartheta)=(\cos \vartheta, \sin \vartheta)^{\mathrm{t}}$ and $\omega^{\perp}(\vartheta)=(-\sin \vartheta, \cos \vartheta)^{\mathrm{t}}$ for $\left.\vartheta \in\right] 0, \pi[$. This parameterization of lines gives rise to the parallel scanning geometry. 
The Radon transform is injective and maps $L^{2}(\Omega)$ boundedly to $L^{2}(Z)$ where $Z:=]-1,1[\times] 0, \pi\left[\right.$ (see e.g. Natterer [14]). Let $\mathbf{R}^{*}: L^{2}(Z) \rightarrow L^{2}(\Omega)$ be the adjoint of $\mathbf{R}$ which is called backprojection. Then,

$$
\mathbf{R}^{*} g(x):=\int_{0}^{\pi} g\left(x^{t} \omega(\vartheta), \vartheta\right) \mathrm{d} \vartheta
$$

Given the tomographic data $g:=\mathbf{R} f$ we wish to reconstruct moments of $f:\left\langle f, e^{i}\right\rangle_{L^{2}(\Omega)}$, $i=1, \ldots, m$, with suitable mollifiers $e^{i} \in L^{2}(\Omega)$. For instance, if $e^{i}$ is an approximation to the $\delta$-distribution located in $x_{i} \in \Omega$ then $\left\langle f, e^{i}\right\rangle_{L^{2}(\Omega)} \approx f\left(x_{i}\right)$.

The computation of the moments is achieved by choosing the mollifiers in the range of $\mathbf{R}^{*}$ :

$$
e^{i}=\mathbf{R}^{*} v^{i} \Rightarrow\left\langle f, e^{i}\right\rangle_{L^{2}(\Omega)}=\left\langle g, v^{i}\right\rangle_{L^{2}(Z)}
$$

(cf. (1.2)). We call $v^{i}$ a reconstruction kernel of $\mathbf{R}$ with respect to $e^{i}$. The mapping $g \mapsto\left(\left\langle g, v^{1}\right\rangle_{L^{2}(Z)}, \ldots,\left\langle g, v^{m}\right\rangle_{L^{2}(Z)}\right)^{\mathrm{t}}$ is then an approximate inverse of $\mathbf{R}$.

In [16] we computed $v^{i}$ from $e^{i}$ where $e^{i} \approx \delta_{x_{i}}$. Here we design the mollifiers from the reconstruction kernels. This is the usual procedure in tomography, (see e.g. Natterer [14, Chapter V]). However, we impose conditions on $v^{i}$ such that $e^{i}$ approximates $\Lambda^{2 m+1} \delta_{x_{i}}, m \in \mathbb{N}_{0}$. For numerical computations, there is no need for an explicit knowledge of the mollifiers.

\section{Obtaining mollifiers from reconstruction kernels}

In this section we study properties of $\mathbf{R}^{*} v$ where $v \in L^{\infty}(\mathbb{R})$ is even, $v(s)=v(-s)$, real-valued, and compactly supported in ] $-1,1[$. These are our standard assumptions on $v$ throughout the paper.

Lemma 3.1. For $x \neq 0$ we have that

$$
\mathbf{R}^{*} v(x)=2 \int_{0}^{\min \{1,\|x\|\}} \frac{v(s)}{\sqrt{\|x\|^{2}-s^{2}}} \mathrm{~d} s
$$

Further, zero moments of $v$,

$$
\int_{-1}^{1} s^{2 k} v(s) \mathrm{d} s=0, \quad k=0, \ldots, m
$$

imply the decay

$$
\left|\mathbf{R}^{*} v(x)\right|=\mathcal{O}\left(\|x\|^{-(2 m+3)}\right) \quad \text { as }\|x\| \rightarrow \infty
$$

Proof. Since $v$ does not depend on the angle $\vartheta$ and is even, we have that

$$
\begin{aligned}
\mathbf{R}^{*} v(r \omega(\varphi)) & =\int_{0}^{\pi} v\left(r \omega(\varphi)^{\mathrm{t}} \omega(\vartheta)\right) \mathrm{d} \vartheta=\frac{1}{2} \int_{0}^{2 \pi} v(r \cos (\varphi-\vartheta)) \mathrm{d} \vartheta \\
& =\int_{0}^{\pi} v(r \cos \vartheta) \mathrm{d} \vartheta
\end{aligned}
$$

We obtain (3.1) by substituting $\cos \vartheta=s / r$ in the last integral above. 
The second assertion follows from (3.1) for $\|x\| \geqslant 1$ using a Taylor expansion of $\left(\|x\|^{2}-s^{2}\right)^{-1 / 2}$ about $s=0$.

In the sequel we denote the Fourier transform of a function $f \in L^{1}\left(\mathbb{R}^{d}\right)$ by $\hat{f}(y):=(2 \pi)^{-d / 2} \int_{\mathbb{R}^{d}} f(x) \mathrm{e}^{-i y^{t} x} \mathrm{~d} x$.

Lemma 3.1 gives that

$$
e:=\mathbf{R}^{*} v \text { is in } L^{1}\left(\mathbb{R}^{2}\right) \cap L^{2}\left(\mathbb{R}^{2}\right)
$$

whenever $v$ has a vanishing mean value, that is, (3.2) holds for $m=0$. Moreover,

$$
\hat{e}(\xi)=\sqrt{2 \pi}\|\xi\|^{-1} \hat{v}(\|\xi\|)
$$

(see e.g. Natterer [14, Chapter II, Theorem 1.4]). Both functions in the above equality are continuous by the Riemann-Lebesgue Lemma.

We have the invariance or intertwining property

$$
\mathbf{R}^{*} \mathscr{V}_{x, \gamma}=\mathscr{U}_{x, \gamma} \mathbf{R}^{*}
$$

for any $x \in \mathbb{R}^{2}$ and $\gamma>0$ where the operators $\mathscr{U}_{x, \gamma}$ and $\mathscr{V}_{x, \gamma}$ are defined by

$$
\mathscr{U}_{x, \gamma} f(z):=f\left(\frac{z-x}{\gamma}\right) \text { and } \mathscr{V}_{x, \gamma} w(s, \vartheta):=w\left(\frac{s-x^{t} \omega(\vartheta)}{\gamma}, \vartheta\right)
$$

Above, $\gamma$ is a scaling or dilation parameter. For $f \in L^{2}(\Omega)$ we define

$$
\mathrm{W}_{e} f(x, \gamma):=\left\langle\mathscr{U}_{x, \gamma} e, f\right\rangle_{L^{2}(\Omega)}
$$

By (3.4) and (3.6), $\mathrm{W}_{e} f$ may be expressed alternatively as

$$
\mathrm{W}_{e} f(x, \gamma)=\left\langle\mathscr{V}_{x, \gamma} v, \mathbf{R} f\right\rangle_{L^{2}(Z)}
$$

which means: $\mathrm{W}_{e} f$ can be computed from the tomographic data $\mathbf{R} f$.

Remark 3.2. Relation (3.5) shows that $e$ from (3.4) is a two-dimensional radial wavelet provided $v$ has a zero mean value. Up to normalization $\mathrm{W}_{e}$ is the corresponding integral wavelet transform (see e.g. [13, Chapter 1.6]).

This connection between the Radon and the wavelet transforms has been observed probably for the first time by Holschneider [7] and has been investigated in detail by Berenstein and Walnut $[1,2]$. Further, Berenstein and Walnut proposed a technique to compute a multiresolution representation of $f$ directly from the Radon data $\mathbf{R} f$. Their results lead to an algorithm for the region-of-interest tomography [15].

Berenstein and Walnut proved a result (Lemma 3.1 in [1]) which is close to our Lemma 3.1. In two space dimensions, however, our decay rates (3.3) are sharper.

We will now investigate the convergence in Sobolev spaces of $\mathrm{W}_{e} f(\cdot, \gamma)$ as $\gamma$ tends to zero. We define the Sobolev spaces $H^{\alpha}\left(\mathbb{R}^{2}\right), \alpha \in \mathbb{R}$, to be the closure of $L^{2}\left(\mathbb{R}^{2}\right)$ with respect to the norm

$$
\|f\|_{\alpha}^{2}:=\int_{\mathbb{R}^{2}}\left(1+\|\xi\|^{2}\right)^{\alpha}|\hat{f}(\xi)|^{2} \mathrm{~d} \xi
$$


The $\Lambda$-operator,

$$
\widehat{\Lambda f}(\xi):=\|\xi\| \widehat{f}(\xi)
$$

maps $H^{\alpha}\left(\mathbb{R}^{2}\right)$ boundedly to $H^{\alpha-1}\left(\mathbb{R}^{2}\right)$.

By $H_{0}^{\alpha}(\Omega) \subset H^{\alpha}\left(\mathbb{R}^{2}\right)$ we denote the closure of $\mathscr{C}_{0}^{\infty}(\Omega)$, the space of infinitely differentiable functions with compact support in $\Omega$, with respect to $\|\cdot\|_{\alpha}$.

The following theorem gives us what we really obtain when computing the moments $\left\langle\mathscr{V}_{x, \gamma} v, \mathbf{R} f\right\rangle_{L^{2}(Z)}$, namely

$$
c\left\langle\mathscr{V}_{x, \gamma} v, \mathbf{R} f\right\rangle_{L^{2}(Z)} \approx \Lambda^{2 m+1} f(x)
$$

where $m \in \mathbb{N}$ depends on $v$ and $c$ is a suitable scaling factor. The above approximation gets better as $\gamma$ becomes smaller.

Theorem 3.3. Let $v \in L^{\infty}(\mathbb{R})$ be even and compactly supported in $]-1,1[$. Further, assume $v$ to have exactly $2 m+1$ zero moments $\left(m \in \mathbb{N}_{0}\right)$, that is, (3.2) holds and

$$
\mu_{m}:=\int_{-1}^{1} s^{2(m+1)} v(s) \mathrm{d} s \neq 0
$$

If $f \in H_{0}^{s}(\Omega), s \geqslant 0$, then

$$
\lim _{\gamma \rightarrow 0}\left\|c_{m} \gamma^{-(2 m+3)} \mathrm{W}_{e} f(\cdot, \gamma)-\Lambda^{2 m+1} f\right\|_{s-(2 m+1)}=0
$$

with

$$
c_{m}=\frac{(-1)^{m+1}(2 m+2) !}{2 \pi \mu_{m}}
$$

Proof. We adapt the proof of Theorem 1.4.2 from [13] to the present situation. With

$$
I(\xi, \gamma):=\left(1+\|\xi\|^{2}\right)^{s-(2 m+1)}\left|c_{m} \gamma^{-(2 m+3)} \widehat{\mathrm{W}_{e} f}(\xi, \gamma)-\|\xi\|^{2 m+1} \hat{f}(\xi)\right|^{2}
$$

we obtain that

$$
\left\|c_{m} \gamma^{-(2 m+3)} \mathrm{W}_{e} f(\cdot, \gamma)-\Lambda^{2 m+1} f\right\|_{s-(2 m+1)}^{2}=\int_{\mathbb{R}^{2}} I(\xi, \gamma) \mathrm{d} \xi
$$

We wish to apply the dominated convergence theorem to the family $\{I(\cdot, \gamma)\}_{\gamma>0}$ of integrable functions.

To this end, we express $\mathbf{W}_{e} f$ by the convolution product $\star$ and by the dilation operator $\mathscr{D}^{\gamma} e(\cdot):=e(\cdot / \gamma)$ :

$$
\mathrm{W}_{e} f(\cdot, \gamma)=\mathscr{D}^{-\gamma} e \star f(\cdot)
$$

Taking the Fourier transform of $\mathrm{W}_{e} f$ with respect to its first variable thus yields

$$
\widehat{\mathrm{W}_{e} f}(\cdot, \gamma)=2 \pi \widehat{\mathscr{D}^{-\gamma} e}(\cdot) \hat{f}(\cdot)=2 \pi \gamma^{2} \mathscr{D}^{-1 / \gamma} \hat{e}(\cdot) \hat{f}(\cdot)
$$


so that

$$
\begin{aligned}
& I(\xi, \gamma)=\left(1+\|\xi\|^{2}\right)^{s-(2 m+1)}|\hat{f}(\xi)|^{2}\left|c_{m} 2 \pi \gamma^{-(2 m+1)} \hat{e}(-\gamma \xi)-\|\xi\|^{2 m+1}\right|^{2} \\
& \stackrel{(3.5)}{=}\left(1+\|\xi\|^{2}\right)^{s-(2 m+1)}|\hat{f}(\xi)|^{2} \\
& \quad \times\left|c_{m}(2 \pi)^{3 / 2} \gamma^{-(2 m+2)} \bar{v}(\gamma\|\xi\|)\|\xi\|^{-1}-\|\xi\|^{2 m+1}\right|^{2}
\end{aligned}
$$

Due to the vanishing moments of $v$, the Taylor expansion of $\hat{v}$ about 0 becomes

$$
\hat{v}(t)=\hat{v}^{(2 m+2)}\left(\tau_{t}\right) t^{2 m+2} /(2 m+2) !
$$

for a suitable $\tau_{t}$ between 0 and $t$. From this Taylor expansion we infer that

$$
I(\xi, \gamma) \leqslant\left(1+\|\xi\|^{2}\right)^{s}|\hat{f}(\xi)|^{2}\left|(2 \pi)^{1 / 2}(-1)^{m+1} \hat{v}^{(2 m+2)}\left(\tau_{\gamma\|\xi\|}\right) / \mu_{m}-1\right|^{2}
$$

By the Riemann-Lebesgue Lemma, the supremum

$$
M:=\sup _{\xi \in \mathbb{R}^{2}}\left|(2 \pi)^{1 / 2}(-1)^{m+1} \hat{v}^{(2 m+2)}\left(\tau_{\gamma\|\xi\|}\right) / \mu_{m}-1\right|^{2}
$$

exists and is independent of $\gamma>0$. Hence, $I(\cdot, \gamma)$ is majorized by an integrable function almost everywhere:

$$
I(\xi, \gamma) \leqslant M\left(1+\|\xi\|^{2}\right)^{s}|\hat{f}(\xi)|^{2}
$$

Since $\tau_{\gamma\|\xi\|}$ goes to zero as $\gamma \rightarrow 0$ and since $\hat{v}^{(2 m+2)}(0)=(-1)^{m+1}(2 \pi)^{-1 / 2} \mu_{m}$ we have pointwise almost everywhere that

$$
\lim _{\gamma \rightarrow 0} I(\cdot, \gamma)=0
$$

The stated convergence follows now from the dominated convergence theorem.

A modification of the proof above shows that the convergence of $\mathbf{W}_{e} f(\cdot, \gamma)$ to $\Lambda^{2 m+1} f$ is quadratic in $\gamma$ with respect to a weaker norm.

Corollary 3.4. Under the assumptions of Theorem 3.3 we have that

$$
\left\|c_{m} \gamma^{-(2 m+3)} \mathrm{W}_{e} f(\cdot, \gamma)-\Lambda^{2 m+1} f\right\|_{s-(2 m+3)} \leqslant C_{m}\|f\|_{s} \gamma^{2}
$$

where

$$
C_{m}=\frac{c_{m}}{\sqrt{2 \pi}(2 m+4) !} \int_{-1}^{1} s^{2 m+4}|v(s)| \mathrm{d} s
$$

Proof. The same arguments as in the proof of Theorem 3.3 verify that

$$
\left\|c_{m} \gamma^{-(2 m+3)} \mathrm{W}_{e} f(\cdot, \gamma)-\Lambda^{2 m+1} f\right\|_{s-(2 m+3)}^{2}=\int_{\mathbb{R}^{2}} I(\xi, \gamma) \mathrm{d} \xi
$$


with

$$
\begin{aligned}
I(\xi, \gamma)= & \left(1+\|\xi\|^{2}\right)^{s-(2 m+3)}|\hat{f}(\xi)|^{2} \\
& \times\left|c_{m}(2 \pi)^{3 / 2} \gamma^{-(2 m+2)} \hat{v}(\gamma\|\xi\|)\|\xi\|^{-1}-\|\xi\|^{2 m+1}\right|^{2}
\end{aligned}
$$

Now, we express $\hat{v}$ in a Taylor expansion of higher order than in (3.9):

$$
\hat{v}(t)=\frac{\hat{v}^{(2 m+2)}(0)}{(2 m+2) !} t^{2 m+2}+\frac{\hat{v}^{(2 m+4)}\left(\tau_{t}\right)}{(2 m+4) !} t^{2 m+4}
$$

for a $\tau_{t}$ between 0 and $t$. (Note that all odd derivatives of $\hat{v}$ vanish at 0 because $v$ is even.) Plugging the latter Taylor expansion into $I(\xi, \gamma)$ yields

$$
I(\xi, \gamma) \leqslant\left(1+\|\xi\|^{2}\right)^{s}|\hat{f}(\xi)|^{2} \frac{\gamma^{4} c_{m}^{2}}{((2 m+4) !)^{2}} \sup _{\tau \in \mathbb{R}}\left|\hat{v}^{(2 m+4)}(\tau)\right|^{2}
$$

Since $\left|\hat{v}^{(2 m+4)}(\tau)\right| \leqslant \int s^{2 s+4}|v(s)| \mathrm{d} s / \sqrt{2 \pi}$ we are done with the proof.

In the remainder of this section we focus on the design of smooth filters $v$ satisfying the hypotheses of Theorem 3.3 and Corollary 3.4. Our main ingredient is an even polynomial $p_{n}$ with multiple zeroes at \pm 1 and a non-vanishing mean value in ]$-1,1\left[\right.$. For instance, let us fix the even polynomial $p_{n}(s):=\left(1-s^{2}\right)^{n}$. With $p_{n}$ and $b, a_{0}, \ldots, a_{m} \in \mathbb{R}$ we define the filter $v_{n, m}$ via

$$
v_{n, m}(s):= \begin{cases}b \prod_{i=0}^{m}\left(s^{2}+a_{i}\right) p_{n}(s) & |s| \leqslant 1 \\ 0 & |s|>1\end{cases}
$$

The constants $b, a_{0}, \ldots, a_{m}$ will be adjusted such that (3.2) holds and $\mu_{m}=(-1)^{m+1}$ $(2 m+2)$ !; see (3.7). Thus, $c_{m}=(2 \pi)^{-1}$ (see (3.8)). We have, e.g., for $|s| \leqslant 1$,

$$
\begin{aligned}
& v_{10,0}(s)=-\frac{1166167275}{1048576}\left(s^{2}-1 / 23\right)\left(1-s^{2}\right)^{10} \\
& v_{10,1}(s)=\frac{3 \cdot 2977529270625}{8388608}\left(s^{2}-1 / 5\right)\left(s^{2}-1 / 45\right)\left(1-s^{2}\right)^{10}
\end{aligned}
$$

Fig. 1 displays the graphs of $v_{10, m}$ as well as the graphs of the radial parts of the related mollifiers $e_{10, m}=\mathbf{R}^{*} v_{10, m}, m=0,1$ (see (3.4) and (3.1)). Note that the $e_{10, i}$ 's are not compactly supported but rapidly decreasing (see (3.3)).

Remark 3.5. The kernels $v_{n, 0}$ have been used before in local tomography. For instance, $K_{1}^{n+1}$ of Faridani et al. [6, Formula (A.18)] and $v_{n, 0}$ coincide. The construction principle for $K_{1}^{n+1}$, which is a little bit different from ours, may be adapted to yield our reconstruction kernels $v_{n, m}$ for general $m$. Essentially, this was already done by Faridani [4, Formula (4.5)]. 

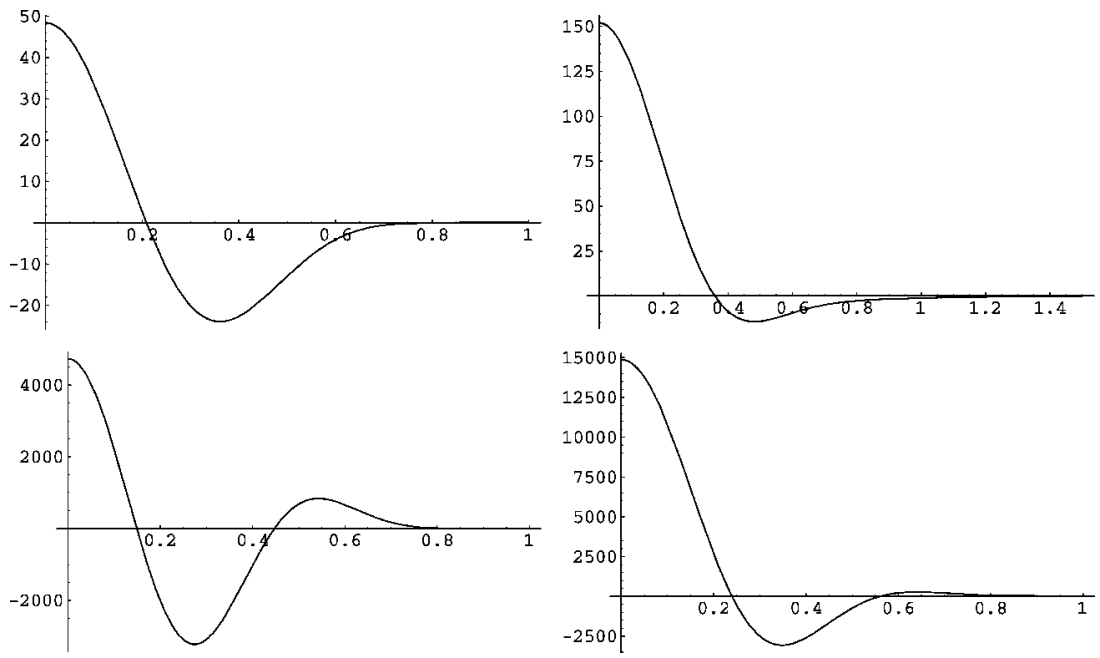

Fig. 1. Reconstruction kernels $v_{10, m}$ (left) and radial parts of the related mollifers $e_{10, m}=\mathbf{R}^{*} v_{10, m}$ (right). Top: $m=0$, bottom: $m=1$. The mollifers are not compactly supported

\section{Filtered backprojection-type algorithm}

In practice only discrete measurements of $\mathbf{R} f$ are available. Given these samples we need to compute an approximation to the inner product $\mathrm{W}_{e} f(x, \gamma)=\left\langle\mathscr{V}_{x, \gamma} v, \mathbf{R} f\right\rangle_{L^{2}(Z)}$.

Assume that we observe $g\left(s_{i}, \vartheta_{j}\right):=\mathbf{R} f\left(s_{i}, \vartheta_{j}\right)$ at $s_{i}=i h_{\mathrm{s}}, i=-q, \ldots, q$, and $\vartheta_{j}=j h_{\vartheta}, j=0, \ldots, p-1$, for $q, p \in \mathbb{N}$ where $h_{\mathrm{s}}=1 / q$ and $h_{\vartheta}=\pi / p$ are the discretization step sizes. Set $h:=\max \left\{h_{\mathrm{s}}, h_{\vartheta}\right\}$.

The trapezoidal rule with abscissæ $\left(s_{i}, \vartheta_{j}\right)$ yields the approximation

$$
\left\langle\mathscr{V}_{x, \gamma} v, \mathbf{R} f\right\rangle_{L^{2}(Z)} \approx T_{h} g(x, \gamma):=\frac{\pi}{q p} \sum_{i=1-q}^{q-1} \sum_{j=0}^{p-1} g\left(s_{i}, \vartheta_{j}\right) v\left(\frac{s_{i}-x^{t} \omega\left(\vartheta_{j}\right)}{\gamma}\right)
$$

The numerical scheme $T_{h} g(x, \gamma)$ is called a filtered backprojection algorithm with filter function $v$ (see e.g. Natterer [14, Chapter V.1]).

The abstract results of [16] formulated in the present context imply the error estimate in Lemma 4.1 below.

Our notation $A \lesssim B$ indicates the existence of a generic constant $c>0$ such that $A \lesssim c B$. In our applications this generic constant will be independent of $f, v, x, h$, and $\gamma$.

Lemma 4.1. Let $f$ be in $H_{0}^{1 / 2+\kappa}(\Omega)$ for a $\left.\left.\kappa \in\right] 0,1\right]$. If $v$ is in $H_{0}^{2}(-1,1)$ and $\left.\left.\gamma \in\right] 0,1\right]$ then

$$
\left\|\mathrm{T}_{h} \mathbf{R} f(\cdot, \gamma)-\mathrm{W}_{e} f(\cdot, \gamma)\right\|_{L^{2}(\Omega)} \lesssim\|v\|_{1+\kappa}\|f\|_{1 / 2+\kappa} \frac{h}{\gamma^{1 / 2+\kappa}}
$$

as $h \rightarrow 0$. The constant involved in the estimate above may depend on $\kappa$. 
Proof. The first part of the proof of Theorem 5.4 in [16] yields

$$
\left|\mathrm{T}_{h} \mathbf{R} f(x, \gamma)-\mathrm{W}_{e} f(x, \gamma)\right| \lesssim\left\|\mathscr{V}_{x, \gamma} v\right\|_{H^{1+\kappa}(Z)}\|f\|_{1 / 2+\kappa} h
$$

By transformation results for Sobolev norms (see e.g. Wloka [21]), we obtain uniformly in $x \in \Omega$ that

$$
\left\|\mathscr{V}_{x, \gamma} v\right\|_{H^{\ell}(Z)} \lesssim\|v\|_{\ell} \gamma^{1 / 2-\ell} \quad \text { for } \gamma \leqslant 1 \quad \text { and } \ell=1,2
$$

Arguments from interpolation theory of Sobolev spaces (see e.g. Lions and Magenes [9]), now lead to

$$
\left\|\mathscr{V}_{x, \gamma} v\right\|_{H^{1+\kappa}(Z)} \leqslant \gamma^{-(1-\kappa) / 2} \gamma^{-3 \kappa / 2}\|v\|_{1+\kappa} .
$$

which, in view of (4.2), allows us to prove the stated error bound.

We are now able to verify convergence of $\mathbf{T}_{h} \mathbf{R} f\left(\cdot, \gamma_{h}\right)$ to $\Lambda^{2 m+1} f$ as $h \rightarrow 0$

Theorem 4.2. Let $v \in H_{0}^{2}(-1,1)$ be as in Theorem 3.3. In particular $v$ has exactly $2 m+1$ zero moments. Choose a $\kappa \in] 0,1]$.

(a) Let $\left\{\gamma_{h}\right\}_{h>0}$ be a family of positive numbers such that

$$
\lim _{h \rightarrow 0} \gamma_{h}=\lim _{h \rightarrow 0} h / \gamma_{h}^{2 m+3.5+\kappa}=0
$$

If $f \in H_{0}^{s}(\Omega)$ for $s \geqslant 2 m+1$ then

$$
\lim _{h \rightarrow 0}\left\|c_{m} \gamma_{h}^{-(2 m+3)} \mathrm{T}_{h} \mathbf{R} f\left(\cdot, \gamma_{h}\right)-\Lambda^{2 m+1} f\right\|_{L^{2}(\Omega)}=0
$$

(b) Let

$$
\gamma_{h}=\mu h^{1 /(2 m+5.5+\kappa)}
$$

where $\mu$ is a positive constant. If $f \in H_{0}^{s}(\Omega)$ for $s \geqslant 2 m+3$ then, as $h \rightarrow 0$,

$$
\left\|c_{m} \gamma_{h}^{-(2 m+3)} \mathrm{T}_{h} \mathbf{R} f\left(\cdot, \gamma_{h}\right)-\Lambda^{2 m+1} f\right\|_{L^{2}(\Omega)} \leqslant\|v\|_{1+\kappa}\|f\|_{s} h^{2 /(2 m+5.5+\kappa)}
$$

Proof. (a) The triangle inequality gives that

$$
\begin{aligned}
& \left\|c_{m} \gamma_{h}^{-(2 m+3)} \mathrm{T}_{h} \mathbf{R} f\left(\cdot, \gamma_{h}\right)-\Lambda^{2 m+1} f\right\|_{L^{2}(\Omega)} \\
& \leqslant\left\|c_{m} \gamma_{h}^{-(2 m+3)} \mathrm{T}_{h} \mathbf{R} f\left(\cdot, \gamma_{h}\right)-c_{m} \gamma_{h}^{-(2 m+3)} \mathrm{W}_{e} f\left(\cdot, \gamma_{h}\right)\right\|_{L^{2}(\Omega)} \\
& \quad+\left\|c_{m} \gamma_{h}^{-(2 m+3)} \mathrm{W}_{e} f\left(\cdot, \gamma_{h}\right)-\Lambda^{2 m+1} f\right\|_{L^{2}\left(\mathbb{R}^{2}\right)} \\
& \quad \leqslant\|\|_{1+\kappa}\|f\|_{1 / 2+\kappa} \frac{h}{\gamma_{h}^{2 m+3.5+\kappa}} \\
& \quad+\left\|c_{m} \gamma_{h}^{-(2 m+3)} \mathrm{W}_{e} f\left(\cdot, \gamma_{h}\right)-\Lambda^{2 m+1} f\right\|_{s-(2 m+1)}
\end{aligned}
$$

The last inequality follows from Lemma 4.1 and from the continuous embedding $H^{s-(2 m+1)}\left(\mathbb{R}^{2}\right) \hookrightarrow L^{2}\left(\mathbb{R}^{2}\right)$ for $s \geqslant 2 m+1$. An application of Theorem 3.3 now verifies the claimed convergence. 
(b) Here, the same estimates used before to prove part (a) lead to

$$
\begin{aligned}
& \left\|c_{m} \gamma_{h}^{-(2 m+3)} \mathrm{T}_{h} \mathbf{R} f\left(\cdot, \gamma_{h}\right)-\Lambda^{2 m+1} f\right\|_{L^{2}(\Omega)} \\
& \lesssim\|v\|_{1+\kappa}\|f\|_{1 / 2+\kappa} \frac{h}{\gamma_{h}^{2 m+3.5+\kappa}} \\
& \quad+\left\|c_{m} \gamma_{h}^{-(2 m+3)} \mathrm{W}_{e} f\left(\cdot, \gamma_{h}\right)-\Lambda^{2 m+1} f\right\|_{s-(2 m+3)} \\
& \lesssim\|v\|_{1+\kappa}\|f\|_{1 / 2+\kappa} \frac{h}{\gamma_{h}^{2 m+3.5+\kappa}}+C_{m}\|f\|_{s} \gamma_{h}^{2}
\end{aligned}
$$

where the last step is due to Corollary 3.4. The proof is finished when balancing the terms on the above right-hand side and taking into account that $C_{m} \lesssim\|v\|_{0}$.

The convergence results of Theorem 4.2 are asymptotic relations in the first place. They do not advise us how to select $\gamma=\gamma_{h}$ for a fixed discretization step size $h$. What is a reasonable value for $\mu$ in (4.4)? The smaller $\mu$ is the faster the filtered backprojection algorithm becomes computationally as the sum over $i$ in (4.1) may be restricted to a few non-zero terms.

The problem of relating $\gamma$ to $h$ was first solved empirically and then analytically explained by Faridani [3], see also Smith and Keinert [18, section VI]. Roughly speaking, choose the smallest possible $\gamma$ such that the discrete kernel $v_{k}=v\left(k \cdot h_{\mathrm{s}} / \gamma\right)$, $k=-q, \ldots, q$, inherits the essential properties from the continuous kernel. These essential properties of $v$ are the moment conditions (see Theorem 3.3).

We propose the strategy $\gamma_{h}:=h_{\mathrm{s}} / s_{\star}=1 /\left(q s_{\star}\right)$ where $s_{\star}$ minimizes locally the discrete moment function $\mathrm{M}_{m}$ in $] 0,1[$ :

$$
\mathrm{M}_{m}(\sigma):=\sum_{\ell=0}^{m}\left|\operatorname{mom}_{\ell}(\sigma)\right| \text { with } \operatorname{mom}_{\ell}(\sigma):=\sum_{k=-\lfloor 1 / \sigma\rfloor}^{\lfloor 1 / \sigma\rfloor} k^{2 \ell} v(k \cdot \sigma)
$$

Here, $\lfloor t\rfloor \in \mathbb{Z}$ for $t \in \mathbb{R}$ denotes the greatest integer: $\lfloor t\rfloor \leqslant t<\lfloor t\rfloor+1$.

Lemma 4.3. Let $v \in \mathscr{C}_{0}^{2 r+2}(\mathbb{R})$ be as in Theorem 3.3 with $2 m+1$ vanishing moments. Assume that $m \leqslant r$. Then,

$$
\mathrm{M}_{m}(\sigma)=\mathcal{O}\left(\sigma^{2(r-m)+1}\right) \quad \text { as } \sigma \rightarrow 0
$$

Proof. We observe that $\sigma^{2 \ell+1} \operatorname{mom}_{\ell}(\sigma)$ is the trapezoidal rule with step size $\sigma$ applied to $\int_{\mathbb{R}} s^{2 \ell} v(s) \mathrm{d} s$. For $0 \leqslant \ell \leqslant m$ we obtain

$$
\sigma^{2 \ell+1} \operatorname{mom}_{\ell}(\sigma)=\mathcal{O}\left(\sigma^{2 r+2}\right) \quad \text { as } \sigma \rightarrow 0
$$

by the summation formula of Euler-Maclaurin (see e.g. Stoer and Bulirsch [19]). Summing up the mom $_{\ell}$ 's to $\mathrm{M}_{m}$ proves (4.5).

In general, $s_{\star}$ is not uniquely determined. Then, we select the largest $s_{\star}$ such that $\mathrm{M}_{m}\left(s_{\star}\right)$ becomes reasonably small. Due to Lemma 4.3 the smoother $v$ is the larger $s_{\star}$ may be chosen. 

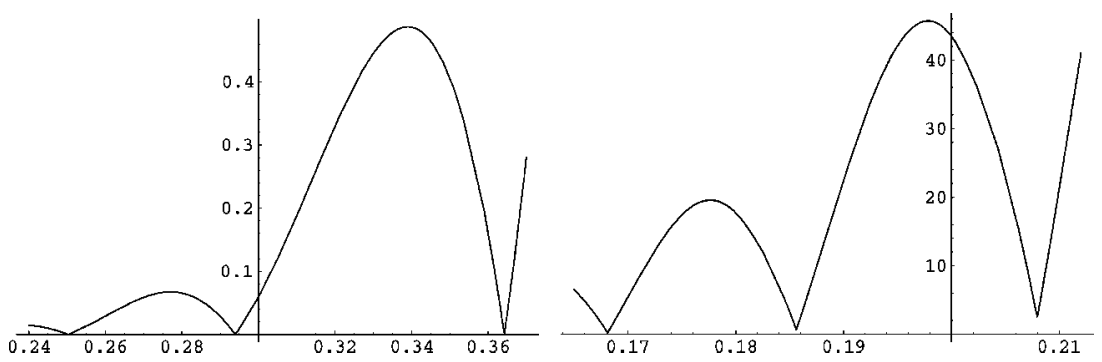

Fig. 2. Discrete moment functions $\mathbf{M}_{0}$ (left) and $\mathbf{M}_{1}$ (right) with respect to $v_{10,0}$ and $v_{10,1}$, respectively

Table 1. Local minima of the discrete moment functions $\mathrm{M}_{0}$ and $\mathrm{M}_{1}$ for values of the argument in Fig. 2

\begin{tabular}{cc}
\hline $\mathrm{M}_{0}$ & $\mathrm{M}_{1}$ \\
\hline 0.3642431314 & 0.2079565234 \\
0.2939686889 & 0.1856192834 \\
0.2503104031 & 0.1681260494 \\
\hline
\end{tabular}

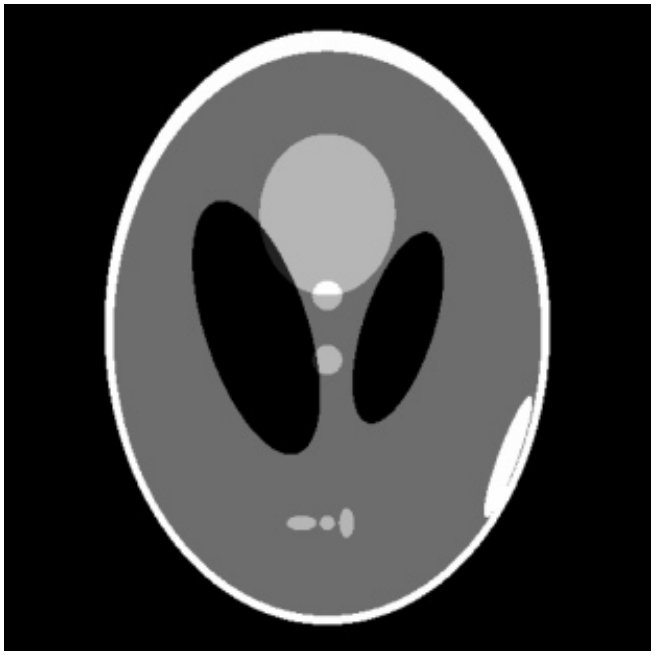

Fig. 3. Head phantom $f_{\text {SL }}$ due to Shepp and Logan [17]

Let us re-consider the two examples $v_{10,0}$ and $v_{10,1}$ from the end of section 3 (see Fig. 1). Fig. 2 shows the graphs of $\mathbf{M}_{0}$ and $\mathbf{M}_{1}$ with respect to $v_{10,0}$ and $v_{10,1}$, respectively, for a meaningful range of $\sigma$. In Table 1 we listed the corresponding local minima $s_{\star}$.

For instance, the largest values $s_{\star, 0}=0.3642431314$ and $s_{\star, 1}=0.2079565234$ of Table 1 lead to the following discrete moments (relative to the kernel maximum):

$$
\sum_{k=-2}^{2} k^{2 \ell} v_{10,0}\left(k \cdot s_{\star, 0}\right) / v_{10,0}(0) \approx \begin{cases}2 \times 10^{-11}, & \ell=0 \\ -1, & \ell=1\end{cases}
$$



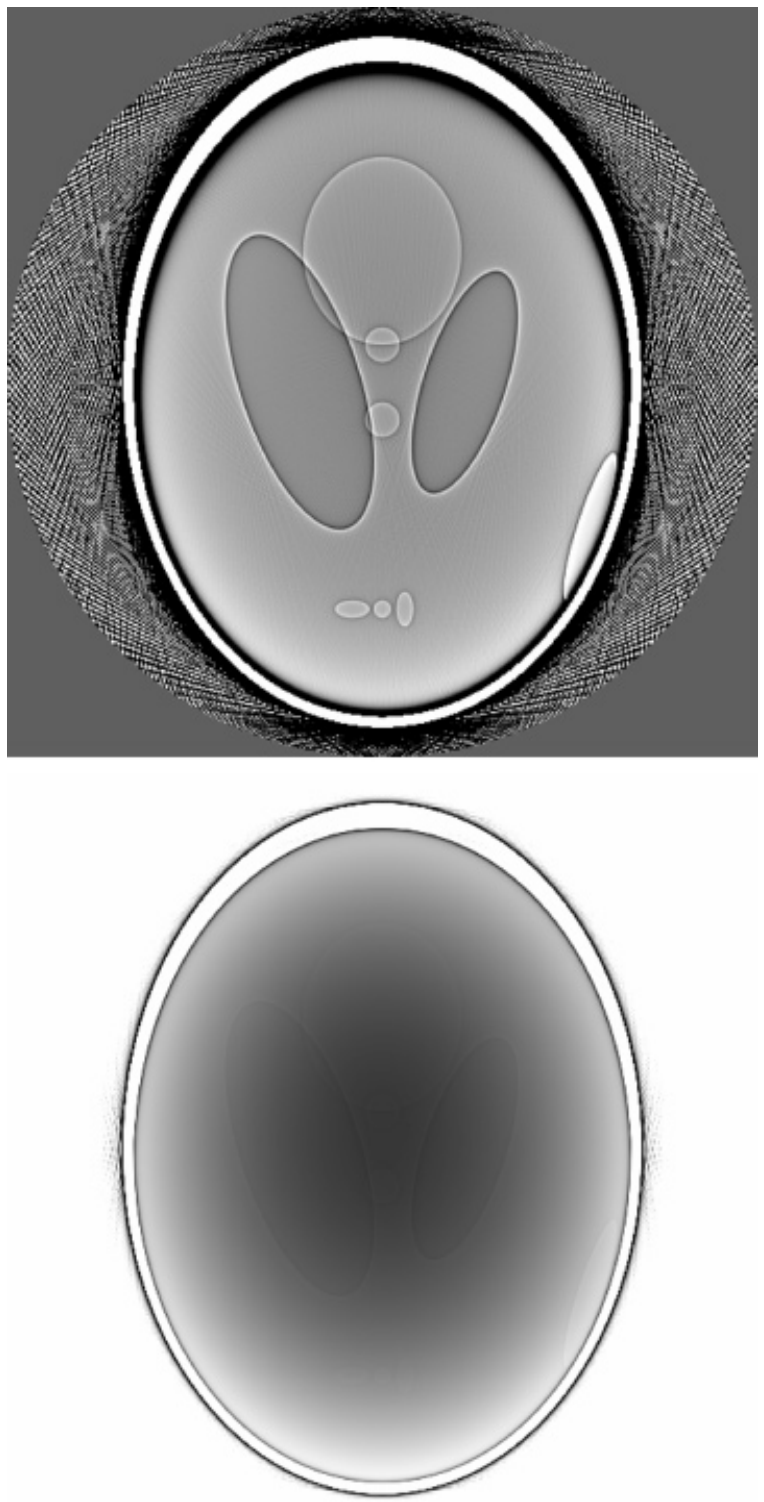

Fig. 4. Reconstruction of $\Lambda f_{\mathrm{SL}}$ by (4.1) with reconstruction kernel $v_{10,0}$ where $\gamma=h_{\mathrm{s}} / 0.3642431314$ (top) and $\gamma=h_{\mathrm{s}} / 0.36$ (bottom)

and

$$
\sum_{k=-4}^{4} k^{2 \ell} v_{10,1}\left(k \cdot s_{\star, 1}\right) / v_{10,1}(0) \approx \begin{cases}-5 \times 10^{-4}, & \ell=0 \\ 2 \times 10^{-11}, & \ell=1 \\ 13, & \ell=2\end{cases}
$$



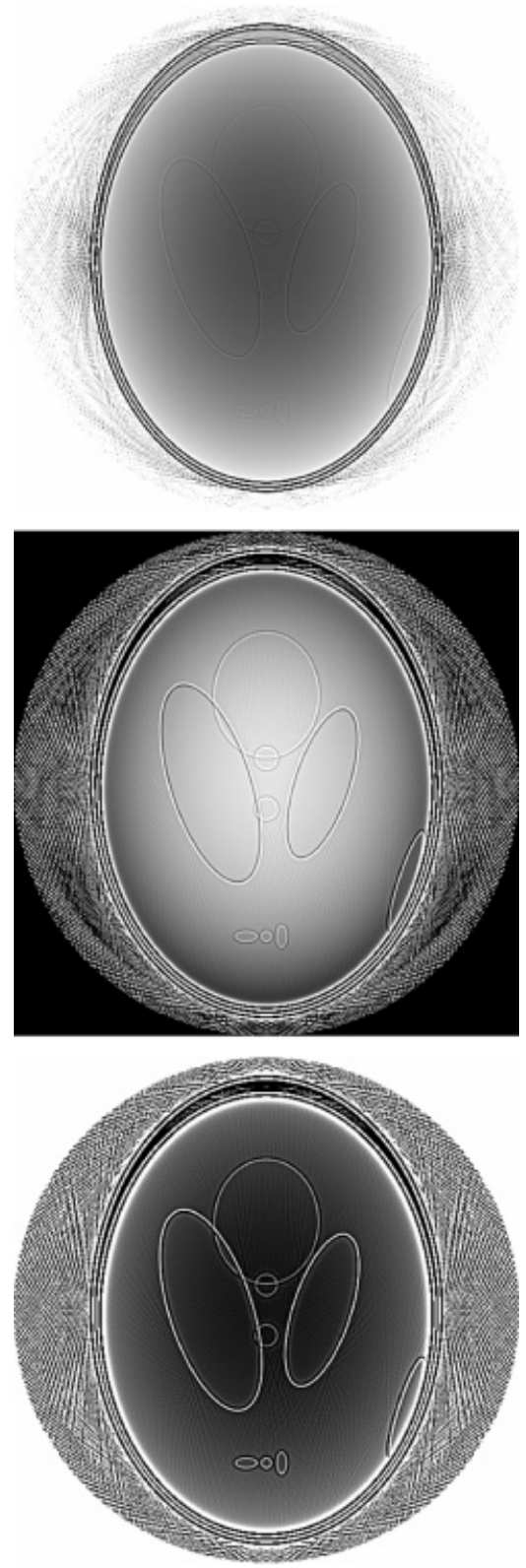

Fig. 5. Reconstruction of $\Lambda^{3} f_{\mathrm{sL}}$ by (4.1) with reconstruction kernel $v_{10,1}$ where $\gamma=h_{\mathrm{s}} / 0.2079565234$ (top), $\gamma=h_{\mathrm{s}} / 0.1856192834$ (middle), and $\gamma=h_{\mathrm{s}} / 0.1681260494$ (bottom)

Please note that we have not been able to prove convergence of $c_{m} \gamma_{h}^{-(2 m+3)} \mathrm{T}_{h} \mathbf{R} f\left(\cdot, \gamma_{h}\right)$ for the strategy $\gamma_{h}=h_{\mathrm{s}} / s_{\star}$ as $h \rightarrow 0$. Indeed, in our numerical experiments we obtained the best reconstructions when choosing $s_{\star}$ dependent on $h$. Here we had to decrease $s_{\star}$ with $h$ which is in accordance with (4.3) and (4.4). 


\section{Numerical experiments}

We demonstrate the mode of action of our strategy for determining $\gamma_{h}$ by local reconstructions. The tomographic data were computed from the phantom designed by Shepp and Logan [17] which simulates the density relations and the geometry in a human skull (see Fig. 3).

Our implementation of the filtered backprojection algorithm (4.1) for the parallel scanning geometry is taken from Natterer [14, Chapter V.1.1]. In all our computations below we worked with $p=400$ directions and 513 rays per direction, that is, $q=256$ and $h_{\mathrm{s}}=1 / 256$, respectively. All reconstructions below are shown on a $511 \times 511$ grid.

Fig. 4 displays two reconstructions of $\Lambda f_{\mathrm{SL}}$ where $f_{\mathrm{SL}}$ denotes the Shepp-Logan phantom from Fig. 3. The reconstruction kernel is $v_{10,0}$ with $\gamma=h_{\mathrm{s}} / 0.3642431314$ (top) and $\gamma=h_{\mathrm{s}} / 0.36$ (bottom). Since 0.3642431314 is a zero of the discrete moment function $\mathrm{M}_{0}$ we obtain a good approximation to $\Lambda f_{\mathrm{SL}}$. However a slight perturbation of this zero yields a catastrophic result. This instability can be seen from Fig. 2. The graph of $\mathrm{M}_{0}$ is very steep near the largest plotted zero. It becomes flatter in the vicinity of smaller zeroes. The latter behaviour was to be expected, see Lemma 4.3. We like to mention that $\gamma=h_{\mathrm{s}} / 0.2939686889$ (cf. Table 1) leads to a reconstruction of $\Lambda f_{\mathrm{SL}}$ which cannot be distinguished visually from the reconstruction with $\gamma=h_{\mathrm{s}}$ / 0.3642431314 .

Finally, we computed approximations to $\Lambda^{3} f_{\mathrm{SL}}$ based on $v_{10,1}$ (see Fig. 5), where $\gamma$ was given by the three local minima of $M_{1}$ from Table 4.1. The reconstruction with the sharpest contrasts belongs to the smallest minimum at which $\mathrm{M}_{1}$ is also smallest.

In our experiments we noticed that the cupping effect is less pronounced when computing $\Lambda^{3} f$ rather than $\Lambda f$. Since $\Lambda^{3} f=-\Lambda \Delta f$ and $\Delta f$ has more vanishing moments than $f$ (at least formally) the observed cup-removing effect of $\Lambda^{3}$ finds its theoretical explanation in the work of Faridani et al. [5, Theorem 5.2].

\section{Acknowledgements}

We thank Adel Faridani and Todd Quinto for their helpful comments on an earlier version of this paper.

\section{References}

1. Berenstein C, Walnut D. Local inversion of the Radon transform in even dimensions using wavelets. In 75 Years of Radon Transform, Gindikin S, Michor P (eds). International Press: Cambridge, MA, 1994; 45-69.

2. Berenstein C, Walnut D. Wavelets and local tomography. In Wavelets in Medicine and Biology, Aldroubi A, Unser M (eds). CRC Press: Boca Raton, FL, 1996; 231-261.

3. Faridani A. Praktische Fragen der lokalen Tomographic. Z. Angew. Math. Mech. 1990; 70: T530-T532.

4. Faridani A. Results, old and new, in computed tomography. In Inverse Problems in Wave Propagation, Chavent G, Papanicolaou G, Sacks P, Symes W (eds). The IMA Volumes in Mathematics and its Applications, vol. 90. Springer: New York, 1997; 167-193.

5. Faridani A, Finch D, Ritman E, Smith KT. Local tomography II. SIAM J. Appl. Math. 1997; 57: 1095-1127.

6. Faridani A, Ritman E, Smith KT. Local tomography. SIAM J. Appl. Math. 1992; 52: 459-484, 1193-1198. 
7. Holschneider M. Inverse Radon transform through inverse wavelet transform. Inverse Problems 1991; 7: 853-861.

8. Katsevich AI, Ramm AG. New methods for finding values of the jumps of a function from its local tomographic data. Inverse Problems, 1995; 11: 1005-1023.

9. Lions JL, Magenes E. Non-Homogeneous Boundary Value Problems and Applications, Vol. 1. Springer: New York, 1972

10. Louis AK. Approximate inverse for linear and some nonlinear problems. Inverse Problems 1996; 12: $175-190$.

11. Louis AK. A unified approach to regularization methods for linear ill-posed problems. Inverse Problems 1999; 15: 489-498.

12. Louis AK, Maass P. A mollifier method for linear operator equations of the first kind. Inverse Problems 1990; 6: 427-440.

13. Louis AK, Maass P, Rieder A. Wavelets: theory and applications. Pure and Applied Mathematics. Wiley: Chichester, 1997.

14. Natterer F. The Mathematics of Computerized Tomography. Wiley: Chichester, 1986.

15. Rashid-Farrokhi F, Liu KJR, Berenstein C, Walnut D. Wavelet-based multiresolution local tomography. IEEE Trans. Image Proc. 1997; 6: 1412-1430.

16. Rieder A, Schuster T. The approximate inverse in action with an application to computerized tomography. SIAM J. Numer. Anal., to appear.

17. Shepp LA, Logan BF. The Fourier reconstruction of a head section. IEEE Trans. Nucl. Sci. 1974; 21: 21-43.

18. Smith KT, Keinert F. Mathematical foundations of computed tomography. Applied Optics 1985; 24: 3950-3957.

19. Stoer J, Bulirsch R. Introduction to Numerical Analysis. Springer: New York, 1996.

20. Vainberg EI, Faingois ML. Increasing the spatial resolution in computerized tomography. In Problems in Tomographic Reconstruction, Alekseev AS, Lavrent'ev MM, Preobrashensky GN (eds). Siberian Branch of the Academy of Science. USSR: Novosibirsk, 1985; 28-35.

21. Wloka J. Partial Differential Equations. Cambridge University Press: Cambridge, U. K., 1987. 